\title{
The SUSY-QCD Coupling Relation
}

\author{
A. Freitas* and P. Z. Skands ${ }^{\dagger}$ \\ *Institut für Theoretische Physik, Universität Zürich, Winterthurerstrasse 190, CH-8057 Zürich \\ ${ }^{\dagger}$ Theoretical Physics, Fermi National Accelerator Laboratory, Batavia, IL 60510-0500, USA
}

\begin{abstract}
In order to establish supersymmetry at future colliders, it is not sufficient to discover new particles, but the identity of gauge couplings and the corresponding Yukawa couplings between gauginos, sfermions and fermions needs to be verified. In detailed studies it was found that the SUSY-Yukawa couplings of the electroweak sector can be studied with great precision at the ILC, but a similar analysis for the Yukawa coupling of the SUSY-QCD sector proves to be far more challenging. Here a first phenomenological study for determining this coupling is presented, using a method which combines information from LHC and ILC.
\end{abstract}

Keywords: Supersymmetry, QCD

PACS: 12.60.Jv, 14.80.Ly

One of the fundamental relations in softly broken supersymmetric theories is the equality between the Yukawa coupling $\hat{g}$ of a gaugino interacting with a fermion and a sfermion and the corresponding Standard Model (SM) gauge coupling $g$ of a gauge boson and two (s)fermions, $g=\hat{g}$. At colliders, this relation can be investigated through the production cross-sections for SUSY particles. Within the Minimal Supersymmetric SM (MSSM), it has been shown [1,2] that the SUSY Yukawa couplings in the electroweak sector can be precisely tested at the per-cent level at a high-energy $e^{+} e^{-}$collider (ILC).

However, the analysis of the SUSY Yukawa coupling $\hat{g}_{\mathrm{s}}$ in the QCD sector is much more difficult. At the ILC this interaction could be studied in the process $e^{+} e^{-} \rightarrow q \tilde{q}^{*} \tilde{g}$, $\bar{q} \tilde{q} \tilde{g}$ [3], but suffers from very low rates and large backgrounds. At the LHC on the other hand, squarks and gluinos with masses below $2-3 \mathrm{TeV}$ are abundantly produced, and their pair production cross sections depend directly on $\hat{g}_{s}$. However, measurements of total cross sections are exceedingly difficult at hadron colliders, with typically only one or two specific decay channels of the squarks and gluinos experimentally accessible [4].

In this contribution, a combination of ILC and LHC measurements is considered, where the relevant branching ratios (BRs) are to be determined at ILC and combined with exclusive cross section measurements in selected channels at the LHC.

In $p p$ collisions, squarks and gluinos can be produced in various combinations, see Fig. 1 . The production of same-sign squarks $\left(\tilde{u}_{\mathrm{L}} \tilde{u}_{\mathrm{L}}, \ldots\right)$ is especially interesting, since it only proceeds through the diagram in the lower left of Fig. 1, and thus depends solely on the SUSY Yukawa coupling $\hat{g}_{s}$. In $p p$ collisions this process dominantly produces $\tilde{u}$ and $\tilde{d}$ squarks, in direct proportion to the quark content of the proton.

While the typically lighter $\tilde{q}_{\mathrm{R}}$ almost exclusively decays directly into the lightest neutralino and a quark jet, thus not allowing charge tagging, the charge of the heavier $\tilde{q}_{\mathrm{L}}$ can be tagged through a chargino decay chain,

$$
\tilde{u}_{\mathrm{L}} \rightarrow d \tilde{\chi}_{1}^{+} \rightarrow d l^{+} v_{l} \tilde{\chi}_{1}^{0}, \quad \tilde{d}_{\mathrm{L}} \rightarrow u \tilde{\chi}_{1}^{-} \rightarrow u l^{-} \bar{v}_{l} \tilde{\chi}_{1}^{0} .
$$



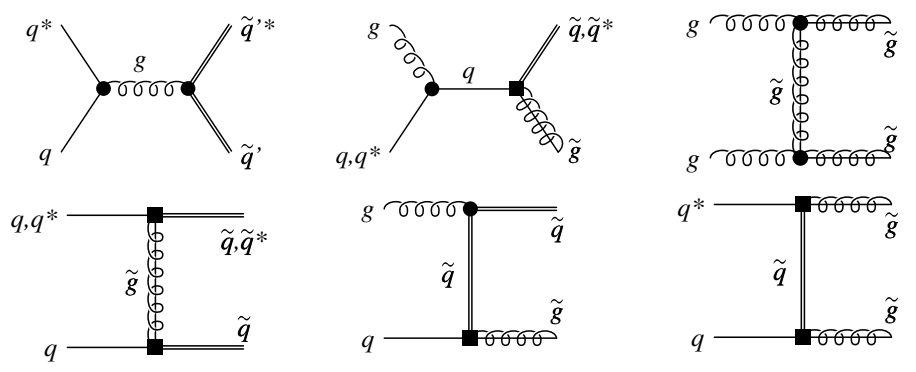

FIGURE 1. Examples for Feynman diagrams for partonic squark and gluino production in $p p$ collisions. Dots indicate the gauge coupling $g_{\mathrm{s}}$, squares the Yukawa coupling $\hat{g}_{\mathrm{s}}$.

The production of same-sign squarks with this decay channel will thus lead to two samesign leptons, two hard jets and missing transverse energy in the final state. In contrast, other direct squark production processes will tend to produce opposite-sign leptons.

A very problematic background can come from gluino pair and mixed gluino-squark production if $m_{\tilde{g}}>m_{\tilde{q}_{\mathrm{L}}}$. In this case, gluinos can decay into quarks and squarks, $\tilde{g} \rightarrow q \tilde{q}_{\mathrm{L}}$, generating a component of two same-sign squarks plus additional jets. This background is very challenging if the mass difference $m_{\tilde{g}}-m_{\tilde{q}_{\mathrm{L}}}$ is small, since then the additional jets from the gluino decay will be soft. In the following we will only consider a scenario where the $m_{\tilde{g}}-m_{\tilde{q}_{\mathrm{L}}}$ mass difference is sufficiently large to allow a veto on additional jets for gluino background reduction. We use a modification of the SPS1a scenario [5], where the gluino mass is raised to $700 \mathrm{GeV}$.

The most important backgrounds from SM sources are $W^{ \pm} W^{ \pm} j j$, where $j$ is a lightflavour jet, and semi-leptonic $t \bar{t}$, with the second lepton coming from the decay of a $b$ quark. Due to the large total $t \bar{t}$ cross section, this can result in a sizable background.

We compute numerical results for expected signal and background levels including some simple estimates for the detector response and resolution (see [6] for details), but do not perform a real experimental analysis. In our scenario, the chargino mainly decays into scalar taus, which subsequently decay into taus. To trace the charge explicitly, here we only consider the leptonic tau BR in the decay chain

$$
\tilde{u}_{\mathrm{L}} \stackrel{65 \%}{\longrightarrow} d \tilde{\chi}_{1}^{+} \stackrel{100 \%}{\longrightarrow} d \tau^{+} v_{\tau} \tilde{\chi}_{1}^{0} \stackrel{35 \%}{\longrightarrow} d \ell^{+}+\not B, \quad \ell=e, \mu,
$$

and similarly for $\tilde{d}_{\mathrm{L}}$. Both signal and top and gluino backgrounds were simulated with PYTHIA 6.326 [7], while the $W W j j$ background was generated with MADEVENT [8]. The cross sections for squark, gluino and top production were normalized with the $K$-factors for next-to-leading order QCD corrections [9], while for the $W^{ \pm} W^{ \pm} j j$ background only leading order results are available.

As a first step, the following preselection cuts are applied: at least $100 \mathrm{GeV}$ transverse missing energy, at least 2 jets with $p_{\mathrm{T}, \mathrm{j}}>100 \mathrm{GeV}$, and two isolated leptons $\ell=e, \mu$ with $p_{\mathrm{T}, \ell}>7 \mathrm{GeV}$. At this level, most backgrounds are still larger than the signal, see Tab 1 . Using a $b$ veto is effective against the gluino and $t \bar{t}$ backgrounds. A high efficiecy of $\varepsilon=90 \%$ reduces the background substantially, at the price of a also high mistagging $D=25 \%$ rate for the signal. The large SM backgrounds can be further suppressed by a cut on the missing transverse energy $Z_{\mathrm{T}}$, with $\#_{\mathrm{T}}>150 \mathrm{GeV}$. At this point, the gluino- 
TABLE 1. Signal and background cross sections for progressive application of cuts.

\begin{tabular}{|c|c|c|c|c|c|c|c|}
\hline Cross Sections & Signal & Backg & ounds & & & & \\
\hline$\sum_{q=u, d, s, c} \sigma(\mathrm{fb})$ & $\tilde{q}_{\mathrm{L}} \tilde{q}_{\mathrm{L}}$ & Sum & $t \bar{t}$ & $W^{ \pm} W^{ \pm} j j$ & $\tilde{q}_{\mathrm{L}} \tilde{g}$ & $\tilde{q}_{\mathrm{L}} \tilde{q}_{\mathrm{L}}^{*}$ & $\tilde{g} \tilde{g}$ \\
\hline Total & 2100 & - & $8 \times 10^{5}$ & - & 7000 & 1350 & 3200 \\
\hline Preselection & 49.2 & 384.6 & 177.7 & - & 136.4 & 23.2 & 47.3 \\
\hline b-veto & 17.1 & 31.4 & 13.0 & - & 10.3 & 7.1 & 1.0 \\
\hline$E_{\mathrm{T}}>150 \mathrm{GeV}$ & 15.1 & 22.2 & 6.1 & - & 9.0 & 6.2 & 0.9 \\
\hline$p_{T, j_{3}}<50 \mathrm{GeV}$ & 7.8 & 5.9 & 2.4 & N/A & 1.0 & 2.5 & 0.03 \\
\hline$p_{T, j_{1}}>200 \mathrm{GeV}$ & 7.0 & $<4.9$ & 1.0 & $<0.7$ & 0.8 & 2.3 & 0.03 \\
\hline
\end{tabular}

related backgrounds dominate. They are reduced by a cut on hard additional jets. By rejecting all events with $p_{\mathrm{T}, \mathrm{j} 3}>50 \mathrm{GeV}$, the ratio of the signal to gluino background is markedly improved. Finally, increasing the transverse momentum cut on the first jet to $p_{\mathrm{T}, \mathrm{j} 1}>200 \mathrm{GeV}$, the top background is suppressed further, resulting in the cross section estimate in Tab. 1. The signal-to-background ratio is 1.4, sufficient to allow a meaningful measurement. With an integrated luminosity of $100 \mathrm{fb}^{-1}$, the statistical error on the same-sign squark cross section is $4.9 \%$.

In order to obtain from the measured rates at the LHC the total squark production cross section, the individual BRs in the decay chain eq. (2) must be determined. Here we explore, how these could be extracted from measurements at ILC.

The chargino BRs can be determined from chargino pair production. Due to the large cross section for that process, all possible chargino decay channels can be easily separated from backgrounds, and the expected error on the BR is about $1 \%$.

In the given scenario the L-squarks are slightly too heavy to be accessible at a $1 \mathrm{TeV}$ linear collider. Here, we instead analyze the production of squarks for a hypothetical $e^{+} e^{-}$collider with a center-of-mass energy of about 1.5 TeV. The L-squarks can decay into the whole spectrum of charginos and neutralinos. While in our scenario the light charginos and neutralinos decay into taus, the heavier states have large BRs into gauge bosons, and can be distinguished through these channels. See [6] for details. Tau leptons can be identified in their hadronic decay mode with roughly $80 \%$ tagging efficiency.

For this work, Monte-Carlo samples for squark pair production in the different squark decay channels have been generated at the parton level with the tools of [2]. Also the most relevant backgrounds have been simulated, stemming from double and triple gauge boson production as well as $t \bar{t}$ production. It is assumed that an integrated luminosity of $500 \mathrm{fb}^{-1}$ is spent for a polarization combination $P\left(e^{+}\right) / P\left(e^{-}\right)=50 \%$ (right) / 80\%(left), which enhances the production cross section both for $\tilde{u}_{\mathrm{L}}$ and $\tilde{d}_{\mathrm{L}}$ production. The BRs are obtained from measuring the cross sections of all accessible decay modes of the squarks and identifying the fraction of decays into one specific decay mode out of these.

Since the squarks are produced in charge-conjugated pairs, it is a priori difficult to distinguish up- and down-squarks in the final state. However, assuming universality between the first two generations, a separation between up- and down-type squarks can be obtained through charm tagging. According to [10], a $c$-tagging efficiency of $40 \%$ is achievable for a purity of $90 \%$. By combining the different decay channels, the following final state signatures are identified as interesting: $j j(n \tau) \not \mathcal{\text { with }} n \in\{1,2,3,4\}$, $c c(n \tau) \not Z$ with $n \in\{2,4\}, j j \tau \tau(Z / W) \not E, c c \tau \tau Z \not Z$, where $j$ indicates an untagged jet, 
TABLE 2. Combination of statistical and systematic errors for the same-sign squark cross section at LHC and the derivation of the strong SUSY-Yukawa coupling.

\begin{tabular}{lrc} 
& $\sigma\left[\tilde{q}_{\mathrm{L}} \tilde{q}_{\mathrm{L}}\right]$ & $\hat{g}_{\mathrm{s}} / g_{\mathrm{s}}$ \\
\hline LHC signal statistics & $4.9 \%$ & $1.3 \%$ \\
SUSY-QCD Yukawa coupling in $\tilde{q}_{\mathrm{L}} \tilde{g}$ background & $2.4 \%$ & $0.6 \%$ \\
PDF uncertainty & $10 \%$ & $2.4 \%$ \\
NNLO corrections & $8 \%$ & $2.0 \%$ \\
Squark mass $\Delta m_{\tilde{q}_{\mathrm{L}}}=9 \mathrm{GeV}$ & $6 \%$ & $1.5 \%$ \\
$\mathrm{BR}\left[\tilde{q}_{\mathrm{L}} \rightarrow q^{\prime} \tilde{\chi}_{1}^{ \pm}\right]$ & $8.2 \%$ & $2.0 \%$ \\
\hline & $17.3 \%$ & $4.1 \%$
\end{tabular}

$c$ a tagged charm jet, and $Z / W$ a hadronically decaying gauge boson. Since several squark decay channels can contribute to most of the final states above, one has to solve a linear equation system in order to derive the individual contributions. With this procedure we estimate the precision for the BRs of the squarks into charginos to be $\mathrm{BR}\left(\tilde{u}_{\mathrm{L}} \rightarrow d \tilde{\chi}_{1}^{+}\right)=(67.7 \pm 3.2) \%$ and $\mathrm{BR}\left(\tilde{d}_{\mathrm{L}} \rightarrow u \tilde{\chi}_{1}^{-}\right)=(63.9 \pm 5.2) \%$.

Based on the simulations for squark production at the LHC and the ILC presented above, one can now derive an estimate for the precision for the determination of the strong SUSY Yukawa coupling $\hat{g}_{s}$. In Tab. 2, the statistical uncertainty is combined with the following systemtic error sources: The remaining background from gluino production at the LHC introduces a systematic error since it depends on $\hat{g}_{\mathrm{s}}$, which is estimated by varying $\hat{g}_{s}$. The uncertainty from the proton parton distribution functions (PDFs) is evaluated by comparing results for different CTEQ PDFs [11]. The uncertainty of the missing $\mathscr{O}\left(\alpha_{\mathrm{s}}^{2}\right)$ radiative corrections are estimated by varying the renormalization scale of the $\mathscr{O}\left(\alpha_{\mathrm{s}}\right)$ corrected cross section within a factor two. Furthermore the cross section depends on the values of the squark masses, which according to [4] can be determined with an error better than $\Delta m_{\tilde{q}_{\mathrm{L}}}=9 \mathrm{GeV}$. Finally, the expected error for the determination of the squark BRs at the linear collider must be included. Combining all error sources in quadrature, it is found that the SUSY-QCD Yukawa coupling $\hat{g}_{\mathrm{s}}$ can be determined with an error $4.1 \%$ in the given scenario.

\section{REFERENCES}

1. J. L. Feng, M. E. Peskin, H. Murayama and X. Tata, Phys. Rev. D 52, 1418 (1995); S. Y. Choi et al., Eur. Phys. J. C 14, 535 (2000); S. Y. Choi, J. Kalinowski, G. Moortgat-Pick and P. M. Zerwas, Eur. Phys. J. C 22, 563 (2001); M. M. Nojiri, K. Fujii and T. Tsukamoto, Phys. Rev. D 54, 6756 (1996); H. C. Cheng, J. L. Feng and N. Polonsky, Phys. Rev. D 57, 152 (1998).

2. A. Freitas, A. v. Manteuffel and P. M. Zerwas, Eur. Phys. J. C 34, 487 (2004); C 40, 435 (2005).

3. A. Brandenburg, M. Maniatis and M. M. Weber, hep-ph/0207278.

4. G. Weiglein et al. [LHC/LC Study Group], hep-ph/0410364.

5. B. C. Allanach et al., Eur. Phys. J. C 25, 113 (2002).

6. A. Freitas and P. Z. Skands, hep-ph/0606121.

7. T. Sjöstrand, S. Mrenna and P. Skands, hep-ph/0603175.

8. F. Maltoni and T. Stelzer, JHEP 0302, 027 (2003).

9. W. Beenakker, R. Höpker, M. Spira and P. M. Zerwas, Nucl. Phys. B 492, 51 (1997).

10. S. M. Xella Hansen et al., LC-PHSM-2003-061 [www-flc.desy . de/l cnotes /].

11. J. Pumplin et al., JHEP 0207, 012 (2002). 